IMAGING TECHNIQUES

\section{Seeing hydrogen}

Determining the behaviour of hydrogen atoms at metal-metal hydride interfaces is central to developing superior hydrogenstorage materials and understanding the deleterious process of hydrogen embrittlement. Yet, owing to its low atomic number, imaging hydrogen - particularly when surrounded by much heavier elements - remains challenging. Now, writing in Science Advances, Sytze de Graaf, Bart J. Kooi and colleagues reveal that a recently developed scanning transmission electron microscopy (STEM) technique can resolve interfacial hydrogen atoms with unprecedented contrast and localization. This demonstration paves the way for studying hydrogen, and other light elements, in crystalline materials.

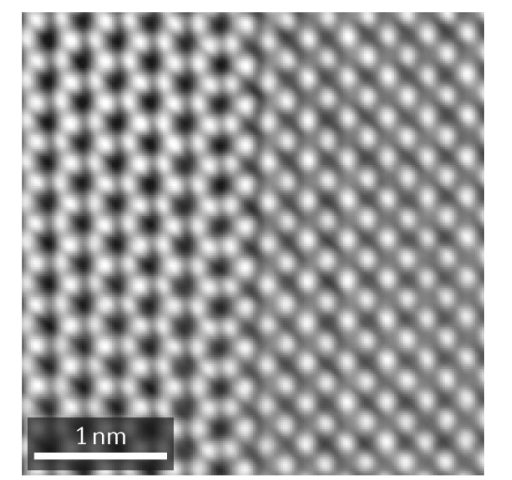

Adapted with permission of AAAS from de Graaf, S. et al. Resolving hydrogen atoms at metal-metal hydride interfaces. Sci. Adv. 6, eaay4312 (2020). $\odot$ The Authors, some rights reserved; exclusive licensee American Association for the Advancement of Science. Distributed under a Creative Commons Attribution NonCommercial License 4.0 (CC BY-NC).
Titanium hydrides readily form at room temperature and are, thus, typically present in samples of the metal. The crystal structure of $\gamma$-TiH and its interface with $\alpha-\mathrm{Ti}$ were widely studied using TEM in the 1980s, but the hydrogen sublattice in $\gamma$-TiH could not be directly imaged. Although three models were proposed to describe the hydrogen atoms at the $\alpha-\mathrm{Ti}-\gamma-\mathrm{TiH}$ interface, it has not been possible to establish which one applies. More than 30 years later, de Graaf, Kooi and co-workers have solved this problem using integrated differential phase contrast (iDPC) STEM.

iDPC uses a segmented detector that analyses the electrons collected within the bright-field region in a physical manner. For thin samples, the contrast in an iDPC image is proportional to the atomic number, increasing the sensitivity of iDPC STEM to light elements. "iDPC images can be directly interpreted, as atom columns appear bright in a dark background," explains de Graaf. "In addition, it has an intrinsic noise-suppression property that enhances the image quality by increasing the signal-to-noise ratio."

The team load bulk titanium with hydrogen through electrochemical polishing and verify the formation of $\gamma$-TiH. Although thin samples are typically required for STEM imaging, thick (30-60 nm) samples were needed to image the $\alpha-\mathrm{Ti}-\gamma-\mathrm{TiH}$ interface. " $\gamma$-TiH is unstable in thin $(<15 \mathrm{~nm})$ regions of the sample," explains de Graaf, "and at intermediate thicknesses (15-30 nm), the large internal stresses that lead to distortion across the interface, prevent crystal alignment." The approach adopted by the team involves rapidly capturing largearea images with a small pixel size. "Certain parts of these images contain well-aligned regions that enable us to image hydrogen at the interface," says de Graaf.

Within the iDPC images, the titanium atoms are well resolved in both $\alpha$-Ti and $\gamma$-TiH. Moreover, within the $\gamma$-TiH crystal, there are clear signals that are consistent with the hydrogen sublattice, enabling the model that describes the $\alpha-\mathrm{Ti}-\gamma-\mathrm{TiH}$ interface to be determined. By contrast, within images captured with more conventional STEM techniques, the hydrogen atoms are either not visualized or the signal-to-noise ratio is too low to reliably ascertain their location. "We've shown that iDPC STEM can image hydrogen almost independent of the thickness and quality of the sample; this is in stark contrast to conventional STEM techniques, which generally require very thin samples with minimal misorientation of the crystal structure to image light elements," says de Graaf.

The robustness of iDPC STEM provides new opportunities for studying technologically relevant materials containing light elements, including lithium and oxygen. Indeed, de Graaf, Kooi and colleagues are already looking to exploit the technique for investigating hydrogen storage in light-metal nanoparticles.

Claire Ashworth

ORIGINAL ARTICLE de Graaf, S. et al. Resolving hydrogen atoms at metal-metal hydride interfaces. Sci. Adv. 6, eaay4312 (2020) 